\title{
AN ELECTROMYOGRAPHIC INVESTIGATION OF SUBLUXATION OF THE PATELla
}

\author{
P. P. MARIANI, I. CARUSO \\ From the Second Orthopaedic Clinic and Dell'Addolorata Hospital, Rome
}

\begin{abstract}
An electromyographic investigation of patients with subluxation of the patella has been carried out on the parts of the extensor apparatus which actively contribute to the alignment of the patella, both before and after the operation to correct this disorder. The electromyographic pictures have revealed a sharp fall in the activity of the vastus medialis, with full recovery to normal values after a corrective operation. Even if the aligning function of the patella is altered by a variety of factors, the present study confirms the importance of the vastus medialis in the pathogenesis of malalignment of the extensor mechanism.
\end{abstract}

In the pathology of the extensor mechanism of the knee, dysplasia of the vastus medialis is often found. Its importance as a factor creating a predisposition to subluxation of the patella has been pointed out repeatedly (Hughston 1968; Fox 1975; Outerbridge and Dunlop 1975; Trillat, Dejour and Puddu 1975; Mansat et al. 1977; Perugia 1977).

Clinical assessment of the functional effectiveness of the vastus medialis and the vastus lateralis, both of which have an aligning function on the patella, is difficult and often of doubtful value. Even the most thorough clinical examination often fails to reveal muscular lesions. Identification is only possible in the gross forms of dislocation or subluxation. Thus an examination of the vastus medialis can sometimes only be carried out during an operation when it becomes possible to identify visually any anomalies present in the muscular fibres or in the insertion of the tendons.

To obtain a better understanding of the role played by the vastus medialis and the vastus lateralis in the alignment of the patella and thus in the pathogenesis of the subluxation, an electromyographic study has been carried out on patients suffering from this disorder, both before and after operation.

\section{MATERIAL AND METHOD}

A preliminary examination of five normal subjects (three men and two women) was performed. This was followed by an examination of eight patients (seven women and one man) suffering from subluxation of the patella which had failed to improve with physiotherapy. All the subjects were aged between twenty and thirty. In the second group an electromyographic investigation was performed before and six to twelve months after the Elmslie-Trillat realignment procedure.

A Medelec MS 6 electromyograph was used. The vastus medialis and vastus lateralis were studied simultaneously in all cases, with unvarying analytical procedures: a current range of 500 microvolts, a graph speed of 2 centimetres per second, and a sweep of 100 milliseconds. The activation of the muscle began with the knee bent at an angle of 90 degrees of flexion, and went through three stages: 90 to 60 degrees, 60 to 30 degrees and 30 degrees to neutral. These were separated by intervals of 15 seconds. The shifts from one position to another were performed slowly, and full extension of the knee was maintained for five seconds.

A Barnet re-education chair was used to allow exact measurement of the angles at which the knee was bent; this offered a low level of resistance, which was held constant throughout the investigation.

\section{RESULTS}

In normal subjects (Fig. 1) the greatest activity of the vastus medialis is recorded between 30 degrees of flexion and the neutral position, and is found at virtually the same levels in the vastus lateralis. A slight difference between the performance of these two muscles is observed only between 90 and 30 degrees, where the

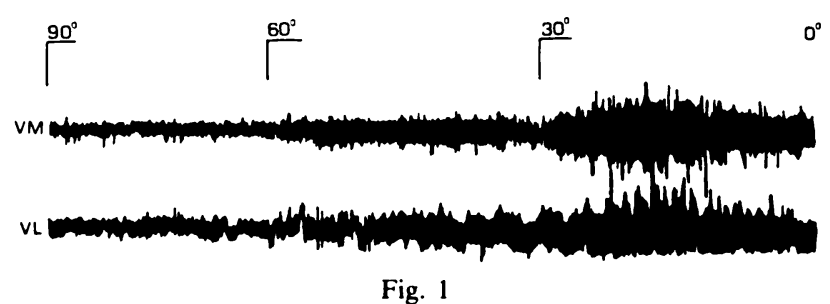

Electromyogram of a control subject. The vastus medialis (VM) has the same activity as that of the vastus lateralis (VL).

vastus lateralis is rather more active. These electromyographic findings were invariable for all the control subjects, some but not all of whom practised competitive sports. The quantitative differences between the measurements obtained with surface electrodes were small and of low significance compared with the tests carried out with needle electrodes.

In seven of the eight patients affected by subluxation of the patella the electromyographic picture of the

P. P. Mariani, M.D., Assistant Professor at the Second Orthopaedic Clinic, University of Rome, Piazzale delle Scienze 11, Rome 00100, Italy. I. Caruso, M.D., Director of the Unit for Functional Recovery and Re-education at the Dell Addolorata Hospital, Via S. Stefano Rotondo 5, Rome (0)184, Italy.

Requests for reprints should be sent to Dr P. P. Mariani. 
vastus medialis obtained before the operation (Fig. 2) showed a marked fall in activity compared with that of the vastus lateralis; this occurred over the whole range of extension but was particularly severe and evident between 30 degrees and zero. In only one case was no difference detected between the performance of the two muscles. The checks carried out on the other leg (Fig. 3), where no abnormality was observed, showed the presence of similar electromyographic changes in the vastus medialis between 30 degrees and zero.

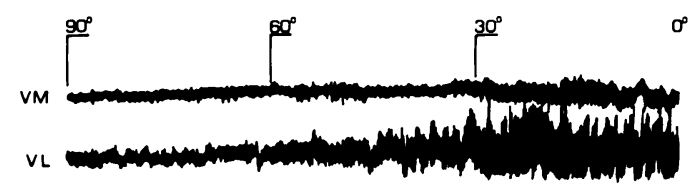

Fig. 2

Electromyogram before operation. A marked fall in the activity of the vastus medialis compared to the vastus lateralis, particularly evident between 30 degrees of flexion and the neutral position ( 0 degrees).
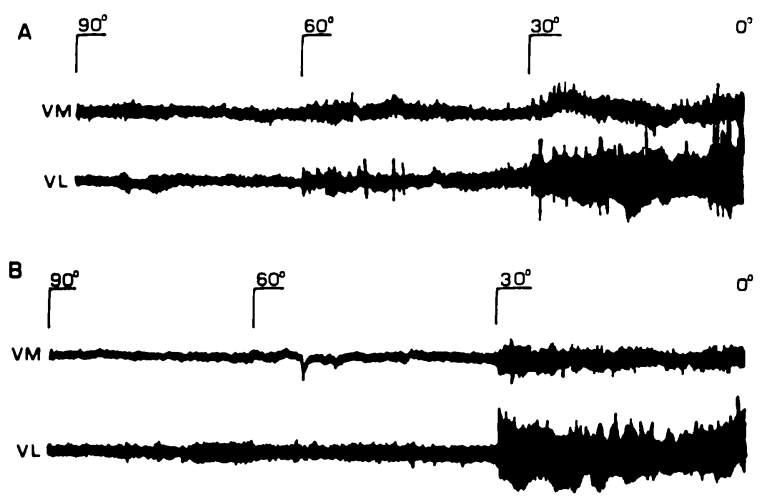

Fig. 3

Electromyogram before operation: A, affected side; B opposite side. A similar fall in the activity of the vastus medialis is seen in both legs.

After the operation to correct the malalignment of the extensor mechanism (Figs. 4 and 5), the activity of the vastus medialis recovered to levels which were clearly higher than those recorded before the operation. In all patients except one, where only a slight improvement was obtained, the performance of the vastus medialis returned to the same levels as those of the vastus lateralis.

\section{DISCUSSION}

Smillie (1962) has called the vastus medialis the "key to the knee", in recognition of its importance in moving the leg through the last 15 degrees.

It is true that this importance has been challenged by some authors (Hallén and Lindahl 1967; Lieb and Perry 1968), but it is certain that the vastus medialis is at its most active during the last phase of extension (Basmajian, Harden and Regenos 1972). Its motor-unit action-potential integrated count, as recorded during a computerised electromyographic study (Lieb and Perry 1971), is about twice that of the other parts of the quadriceps at this stage. No precise answer to Basmajian's query, "Why are there four heads for a muscle?" can yet be given.

Thus, even if the activity of the vastus medialis during the extension of the knee is still a controversial issue, there is at least unanimous agreement that the vastus medialis prevents lateral dislocation of the patella, by resisting the subluxatory pressures created by valgus angulation of the knee and by the vastus lateralis (Lieb and Perry 1968, 1971; Basmajian (t al. 1972). Any change, whether congenital or post-traumatic. in the muscular balance between vastus medialis and vastus lateralis is bound to affect the patellofemoral joint, giving rise, according to the severity of the disorder, to a variety of clinical presentations.

This finding seemed to be confirmed by the electromyographic findings, which showed that in seven of the eight patients with subluxation of the patella there

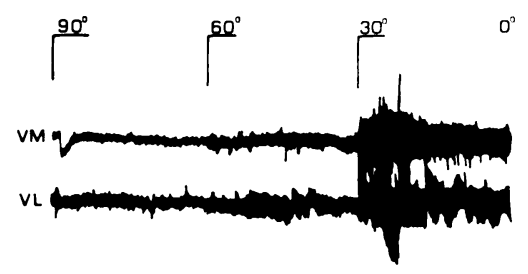

Fig. 4

Electromyogram after operation. The activity of the vastus medialis rises to the same levels as those of the vastus lateralis.
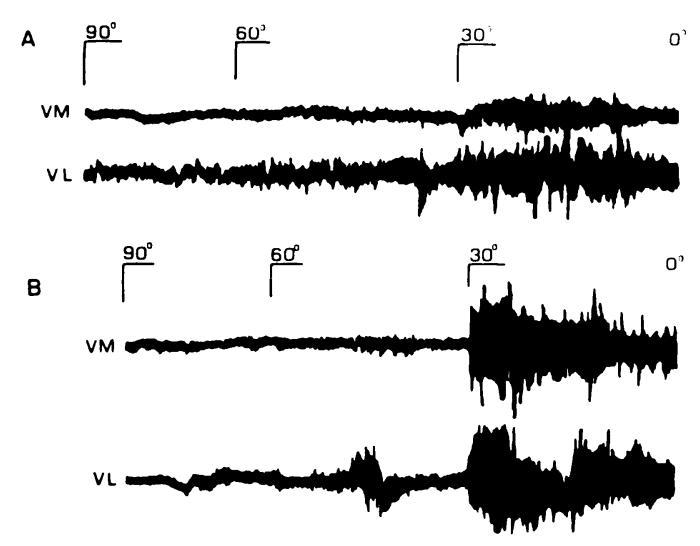

Fig. 5

Electromyogram before (A) and after (B) operation. showing the increase in activity of the vastus medialis.

was a sharp fall in the range and motor-unit actionpotential of the vastus medialis throughout its range of extension, especially during the last 30 degrees. Moreover, the presence of similar electromyographic changes in the other leg, which presented no clinical features, showed that such changes were not attributable to muscle wasting, but to the operation of factors, usually active in both legs, which predisposed a subject 
to subluxation of the patella. Independent confirmation of this was provided by the only case in which no electromyographic changes were recorded; this showed gross wasting of the quadriceps and such acute symptoms of pain that the subject had to stop playing sports.

Another conclusion that may be drawn from the electromyographic findings after the operation is that the Elmslic-Trillat method, by acting on the insertion of the patellar tendon and on the lateral retinacula, is able to correct muscular malalignment of the extensor mechanism. It therefore seems that dysplasia of the vastus medialis is a secondary event, consequent on previous static alterations in the extensor mechanism-a rise in the $\mathrm{Q}$ angle, a high patella, genu recurvatum and proximal valgus (bayonet) deformity of the tibia. We do not wish to assert that such a conclusion is correct, but our findings do allow us to hypothesise that functional insufficiency of the vastus medialis tends to aggravate such static alterations, so setting up a vicious circle. We believe that muscular malalignment, whether primary or secondary, is an invariable and extremely important feature in the pathogenesis of subluxation of the patella. This feature must, therefore, be assessed and borne in mind during physiotherapy or operation.

The authors are most grateful to Professor 1 . S. Smillie for his advice and encouragement during the preparation of this paper.

\section{REFERENCES}

Basmajian, J. V., Harden, T. P., and Regenos, E. M. (1972) Integrated actions of the four heads of quadriceps femoris: an electromyographic study. Anatomical Record, 172, 15-20.

Fox, T. A. (1975) Dysplasia of the quadriceps mechanism. Surgical Clinics of North America, 55, 1, 199-226.

Hallén, L. G., and Lindahl, O. (1967) Muscle function in knee extension. Acta Orthopaedica Scandinavica, 38, 434-444.

Hughston, J. C. (1968) Subluxation of the patella. Journal of Bone and Joint Surgery, 50-A, 1003-1026.

Lieb, F. J., and Perry, J. (1968) Ouadriceps function: an anatomical and mechanical study using amputated limbs. Journal of Bone and Joint Surgerv, 50-A, $1.535-1.548$

Lieb, F. J., and Perry, J. (1971) Quadriceps function: an electromyographic study under isometric conditions. Journal of Bone and Joint Surgery: 53-A, 749-758.

Mansat, C., Duboureau, L., Cha, P., and Dorbes, R. (1977) Déséquilibre roiulien et instabilité rotatoire externe du genou. Revue du Rhumatisme et des Maladies Ostéo-articulaires, 44, 115-123.

Outerbridge, R. E., and Dunlop, J. A. Y. (1975) The problem of chondromalacia patellac. Clinical Orthopaedics and Related Research, $110,177-196$.

Perugia, L. (1977) La patologia del meccanismo estensore. Read at the International Meeting of the Society of Knee Surgery, Rome.

Smillie, I. S. (1962) Injurie's of the Knee Joint. Third edition, pp. 3-5, 99. Edinburgh and London: E. \& S. Livingstone.

Trillat, A., Dejour, R., and Puddu, G. C. (1975) La sublussazione recidivante della rotula. Giornale Italiano Ortopedia e Traumatologia, 1, 209-219. 